\title{
Emotionally Intelligent Chatbot
}

\author{
Shubham Kokane ${ }^{1}$, Shreeyash Khalate ${ }^{2}$, Shre ya Newale ${ }^{3}$, Sakshi \\ Dube war $^{4}$, Jameer Kotwal ${ }^{5}$ \\ ${ }^{1,2,3,4}$ B.E (Computer Engineering), Pimpri Chinchwad College Of Engineering And Research, \\ Ravet, Pune, Maharashtra, India \\ ${ }^{5}$ Asst. Professor, Department of Computer Engineering, Pimpri Chinchwad College Of \\ Engineering And Research, Ravet, Pune, Maharashtra, India,
${ }^{1}$ Email: shubhamkkn7@gmail.com 2Email:shreekhalate555@gmail.com ${ }^{3}$ Email:shreyanewale11@gmail.com ${ }^{4}$ Email:sakshidubewar@gmail.com
${ }^{5}$ Email: jameer.kotwal@pccoer.in

\begin{abstract}
An emotionally intelligent chatbot system aims to make an effective conversation between humans and machines in as natural and interactive manner as possible. The chatbot agent has pre-embedded knowledge base to identify the sentences, intents, entities and context of the input query to be precise for making a valid, predictable decision itself as a self-generated response to answer the query. The present technical project consists of developing an intelligent system for college enquiry purposes using a web-based chatbot agent, through machine learning, query processing and sentiment and emotion classification system to analyze the sentiment of the visitor towards the college.

Emotionally Intelligent College Enquiry Chatbot System is nothing but chatbot to understand the user queries and respond to it during a conversation. Chatbot can actively help human to involve in a digital automated conversation with a machine or a system with effective. In the following proposed system, feature extraction and data cleaning techniques are applied on the dataset and classifiers such as multinomial naive bayes, logistic regression and $k$ nearest neighbors are used to train the model. The classifier with highest accuracy is further used for emotion classification of users.
\end{abstract}

Keywords: Emotion Classification, Sentiment Analysis, Multinomial Naive Bayes, NLTK, TextBlob

\section{Introduction}

Textual conversational agents or chatbots development gather tremendous traction from both academic and industries in recent years. Nowadays, chatbots are widely used as an agent to communicate with a human in some services such as booking assistant, customer service and also a personal partner. Emotion Recognition is a buzz research topic in the field of Human Computer Interaction. It has potentially wide applications, such as the interface with robots, banking, call centers, car board systems, computer games etc. Many chatbot systems are created for various purposes but very few of them are designed to know the feedback of the system and the organization indirectly from the user. That is why the Emotionally Intelligent College Enquiry Chatbot System came into picture in our research work.

The proposed idea consists of developing an intelligent chatbot system for college enquiry purposes using a web-based chatbot agent platform DialogFlow, generating a valid response to the user, and retrieving the conversation history from google logfiles for sentiment and emotion classification of the user to determine his/her interests in the college towards taking the admission. Human sentiment can be measured in scores between -1 and 1 which is also called the polarity of the text. For detailed emotion recognition apart from Positive, Negative or Neutral sentiments, we have used the twitter 
dataset which contains tweets with corresponding emotions.

The dataset has 40,000 rows and three classifiers as Multinomial Naive Bayes, Logistic Regression and K-Nearest Neighbors are trained. The models are evaluated on their accuracy, f1 score and confusion matrices and the most accurate one is selected. Furthermore, an emotion classific ation is carried out on the conversation his tory of users in general and each query will be classified into seven different emotional classes such as neutral, happy, excited, satisfied, not satisfied, boredom and disgust and in the results dataset each query is attached with a polarity, analyzed sentiment and corresponding emotion for more details of the affective states of the user.

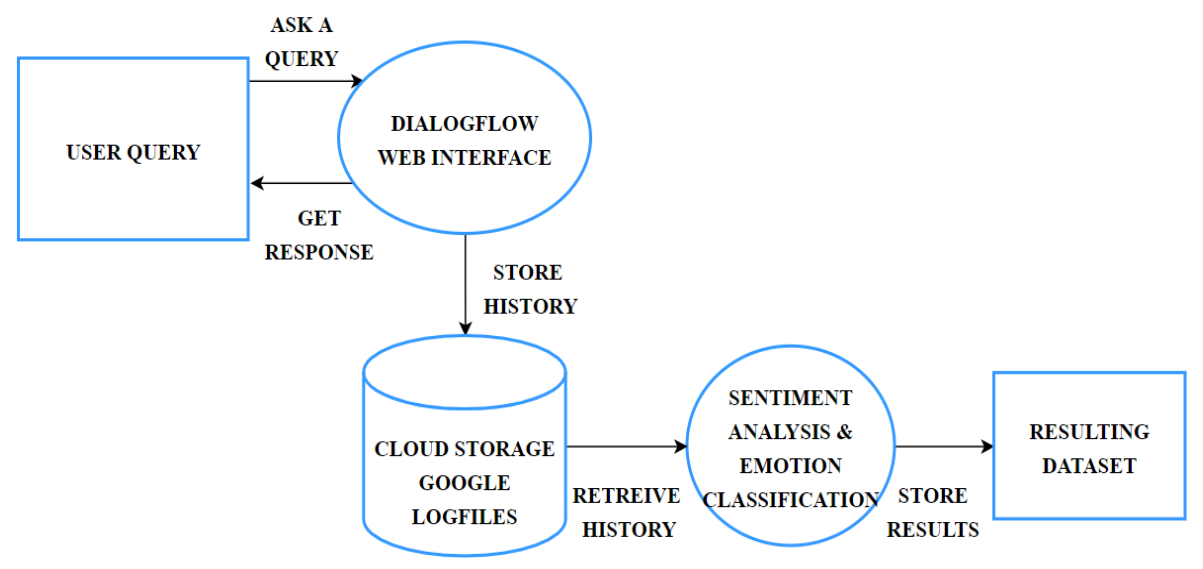

Figure 1. Basis of Chatbot System

\section{Literature Review}

\section{Ms. Ch. Lavanya Susanna, R. Pratyusha, P. Swathi, P. Rishi Krishna, V. Sai Pradeep," COLLEGE ENQUIRY CHATBOT", International Research Journal of Engineering and Technology (IRJET), on 2020 \\ This paper proposes to develop an algorithm which will be wont to identify answers associated with user submitted questions. To develop a database were all the related data are going to be stored and to develop an internet interface. The user will not waste a lot of time searching for the acceptable notices.}

\section{A. Harimi1*, A. Shahzadi1, A.R. Ahmadyfard and Kh. Yaghmaie Department of Electrical Engineering and Robotics, Shahrood University of technology, Iran, 09 February 2013 \\ This paper proposes to evaluate the proposed SPs and HEs for the recognition of human emotions from speech. Aim of a speech emotion recognition system is to recognize emotions for real work data, evaluating the proposed system under different conditions such as the presence of noise and chatter is useful.}

Sumit Wailthare, Tushar Gaikwad, Ketkee Khadse, Pooja Dubey" Artificial Intelligence Based Chat-Bot", Dept. Of Computer Science Technology, Nagpur Institute of Technology, Nagpur, Maharashtra, India 03 (IRJET), Mar-2018 This paper has to be providing answers to the query of the user very effectively. The objective of the project is to develop an algorithm that will be used to identify answers related to user submitted questions. The need is to develop a database where all the related data will be stored and to develop a web interface.

Gustavo Assunção, Paulo Menezes Institute of Systems and Robotics, Coimbra, Portugal," Speak er Awareness for Speech Emotion Recognition" (2020)

In this paper, we evaluated a large-scale machine learning model for classific ation of emotional states trained for speaker identification which aim to verify that SER improves when some speaker's emotional prosody cues are considered. 


\section{Methodology}

The machine-based emotion classification of the user has gained a lot of attention in recent times especially by the social media companies for businesses such as ad recommendations, etc. The modules used in this methodology are chatbot design, conversation history retrieval, data pre-processing, feature engineering, sentiment analysis, emotion classification and exploratory data analysis. The proposed methodology is shown in Figure. 2

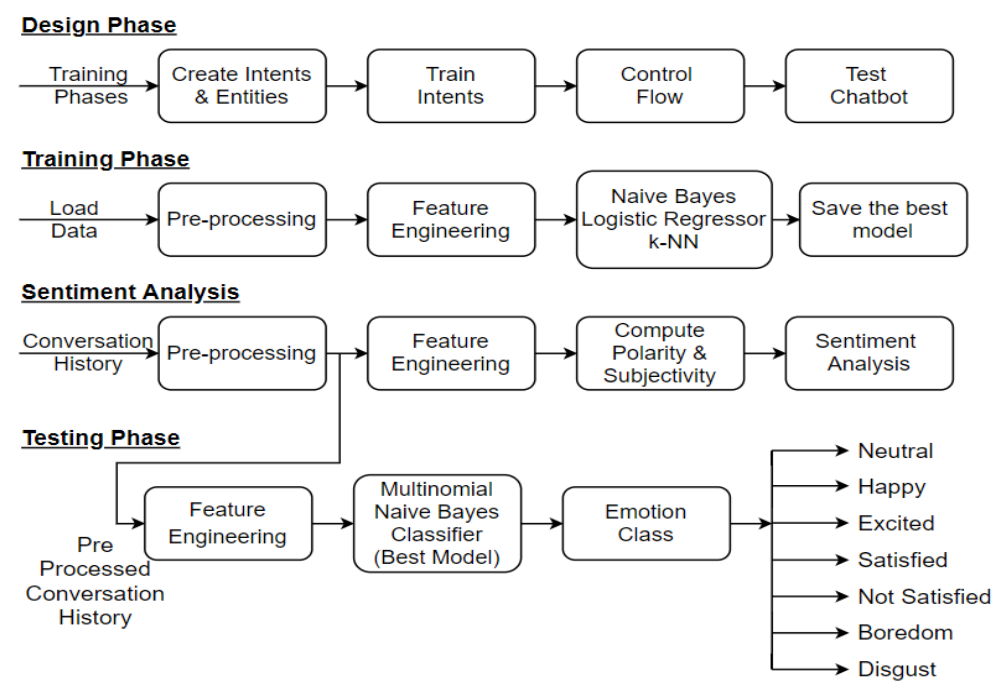

Figure 2. System Architecture

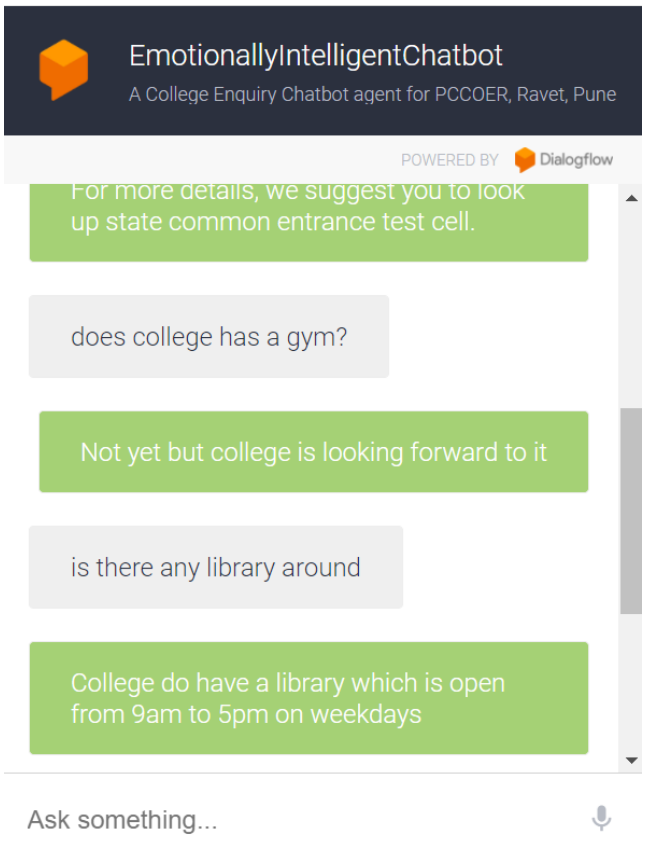

Figure 3. Chatbot Interface

\section{1: Data Pre-processing}

Data preprocessing is a technique in which we transform the raw data in a useful, clean and efficient format for further training the models. In our module, we transform the raw conversation history downloaded from google $\log$ files into clean data. Initially it contained 366 rows and 171 columns and only 2 were extracted 'textPayload' and 'timestamp' for our purposes. All the rows containing null values in the 'textPayload' column are dropped and the data frame is ready for the sentiment analysis part. On the other hand, we loaded a preprocessed twitter emotion dataset for the training phase (for 
training model for emotion classification) as shown in Figure 2 which contained 40000 rows and 5 columns from which 2 columns 'emotion' and 'content' are retrieved for further purpose. The tweets are cleaned using regular expressions for removing mentions, hashtags, retweets, hyperlinks and punctuation marks. Here lemmatization is introduced. Lemmatization is a process of converting all the words in the 'content' column to their base form for increasing the accuracy of the model. An NLTK toolkit is used for removing stopwords from the twitter emotion data and lemmatization is applied to the 'content' column.

\subsection{Feature Engineering}

Feature engineering is a process of transforming the features in the dataset into formats that algorithms can understand that better represent the input values to the predictive models. Here we use the Countvectorizer from scikit-learn for feature extraction. We transform the twitter data into vectors based on the frequency count of each word in the text in the 'content' column. CountVectorizer creates a matrix in which each unique word is represented by a column of the matrix, and each tweet sample from the 'content' column is a row in the matrix. The conversation history is split into two data frames for query requests and chatbot responses for further analysis.

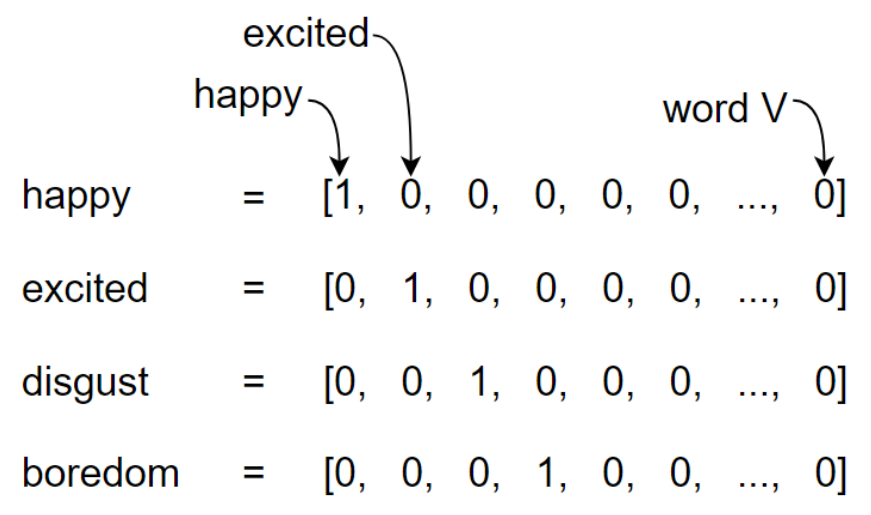

Count Vectorization

Figure 4. Count Vectorization Example

\subsection{Sentiment Analysis}

Sentiment analysis is a process of analyzing the emotional sentiment in the text. Here, we compute the polarity and subjectivity of the text using TextBlob and visualize them with scatter plots and histograms (as shown in Figure. 5 and Figure. 6). The sentiments are classified in three classes as Positive, Negative and Neutral sentiment. These are computed based on the polarity of the text which defines the orientation of the sentiment. The polarity lies in the range of $[-1,1]$. Polarity score below 0 is considered as negative sentiment, polarity above 0 reflects the positive sentiment and polarity equal to 0 show $\mathrm{s}$ neutral sentiment. 


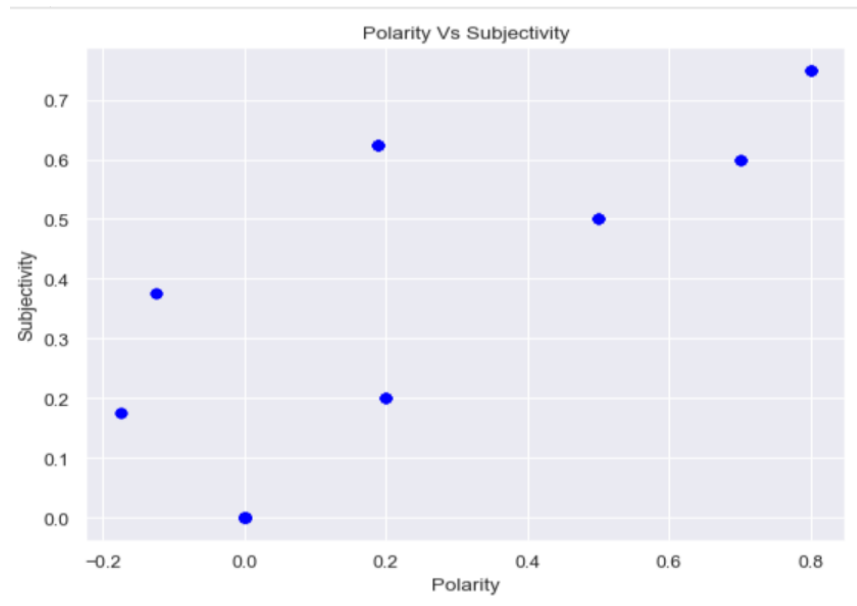

Figure 5. Polarity vs Subjectivity

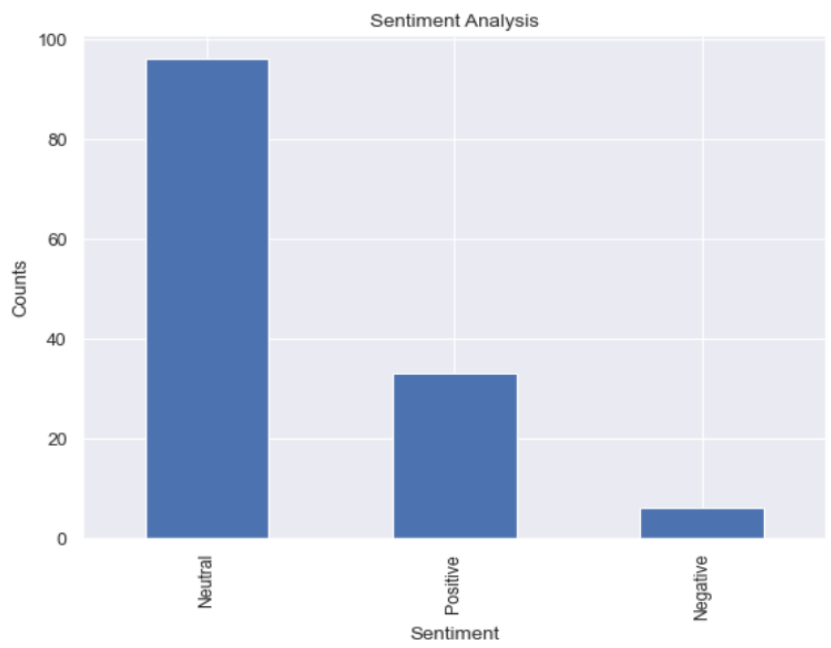

Figure 6. Sentiment Analysis

\subsection{Multinomial Naive Bayes Classification}

Multinomial Naive Bayes is a probabilistic learning method based on Bayes theorem which predicts the tag of a text (in our case 'content' column data) by computing the probability of each tag for a given sample and then gives the tag with highest probability as output. Bayes theorem calculates the probability of a word occurring in the text based on the prior knowledge of conditions related to occurrence of that word in the training dataset. It is based on the following equation.

$$
P(A \mid B)=\frac{P(B \mid A) \times P(A)}{P(B)}
$$

Where we are calculating the probability of class A when predictor B is already provided.

$\mathrm{P}(\mathrm{B})=$ Probability of class $\mathrm{B}$

$\mathrm{P}(\mathrm{A})=$ Probability of class A

$\mathrm{P}(\mathrm{B} \mid \mathrm{A})=$ Occurrence of predictor $\mathrm{B}$ given class A probability

The vectors formed from count vectorizer and the target variable are trained with Multinomial Naive Bayes classifier along with Logistic Regressor and k-NN classifier. We have implemented Naive Bayes with the following steps: 
1) Take the input from the count vectorizer.

2) Take the input from the target column 'emotion'.

3) Train the model.

4) Comparison of the features from test data to training data is done in following steps for classifying the emotion in the test data:

1. Naive Bayes creates a frequency table of training data and lists count of all the words against corresponding emotion.

2. It finds probability of each word for each emotion and creates a likelihood table.

3. It assumes that the effect of predictor (A) on a given class (B) is independent of the values of other predictors.

4. It computes the posterior probability for each emotion using the Naive Bayes theorem.

5. The emotion with the highest probability will be the outcome of that word in the data.

6. Classified emotion of the string is returned.

5) Repeat steps from 1 to 4 until all the training set is computed.

\section{Results and Discussion}

We have used the twitter emotion dataset from Kaggle to evaluate the performance of our emotion classification system model. The dataset (after cleaning) is shown in the Figure. 7 below.

\begin{tabular}{rrr} 
& emotion & content \\
\hline 0 & neutral & know listenin bad habit earlier started freaki... \\
1 & not satisfied & layin n bed headache ughh waitin call \\
2 & not satisfied & funeral ceremony gloomy friday \\
3 & excited & want hang friend soon \\
4 & neutral & want trade someone houston ticket one \\
$\ldots$ & $\ldots$ & \\
39995 & neutral & \\
39996 & satisfied & happy mother day love \\
39997 & satisfied & happy mother day mommy woman man long momma so... \\
39998 & happy & wassup beautiful follow peep new hit single ww... \\
39999 & satisfied & bullet train tokyo gf visiting japan since thu... \\
40000 & rows $\times 2$ columns &
\end{tabular}

Figure 7. Cleaned Twitter Emotion Dataset

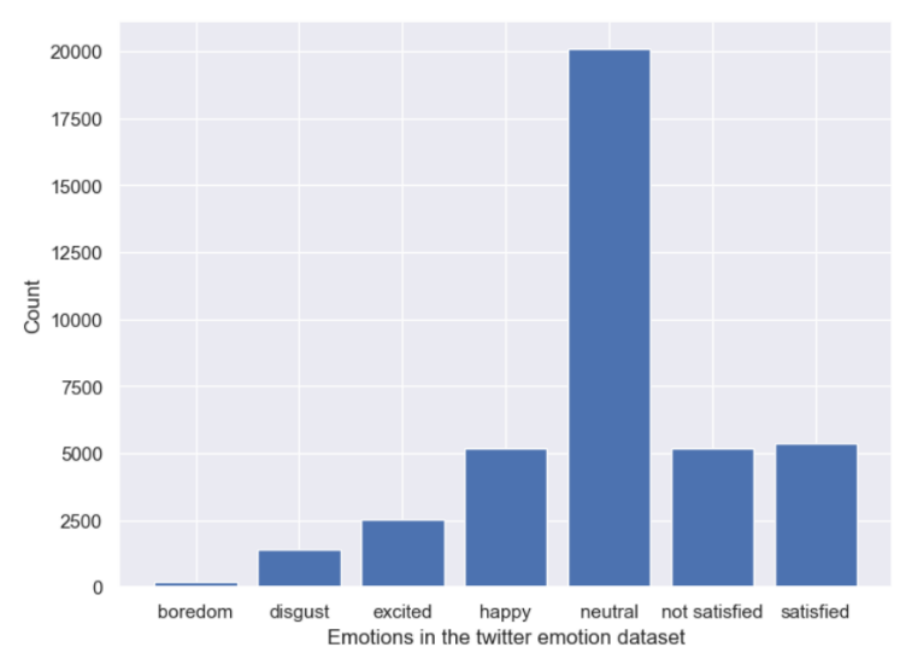

Figure 8. Emotions in the Twitter Dataset 
The results obtained from our proposed system are explained below. The overall performance of the system is evaluated by Accuracy and F1 score. Accuracy is calculated by:

Accuracy $=\frac{\text { Number of emotions correctly recognized }}{\text { Total number of emotions }} \times 100$

The accuracy of the proposed system is then calculated by using the total number of correctly classified emotions divided by the total number of emotions. The accuracy for logistic regressor is $71.5 \%$, and of $\mathrm{k}-\mathrm{NN}$ is $59 \%$, and maximum accuracy came out to be of naive bayes i.e. $73.8 \%$. The confusion matrix is evaluated for the naive bayes model and heatmap is generated for that confusion matrix displayed by percentages as shown below in Figure. 9

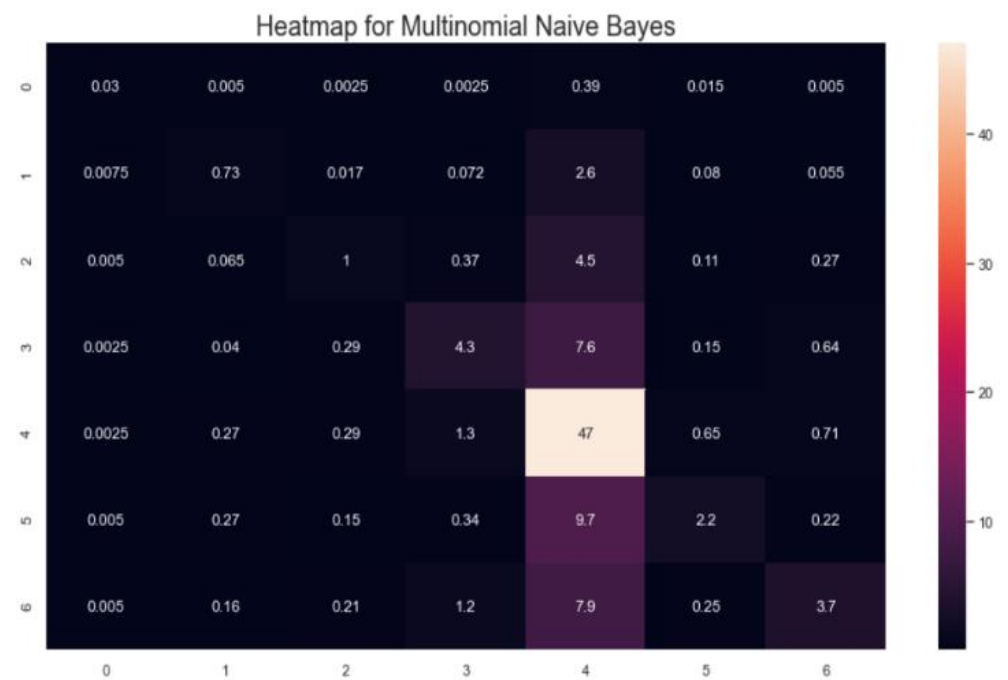

Figure 9. Confusion Matrix of the Proposed System for Multinomial Naive Bayes Classifier

The $\mathrm{F} 1$ score of logistic regressor is obtained as $59.7 \%$, that of $\mathrm{k}-\mathrm{NN}$ is observed as $35.08 \%$ and of multinomial naive bayes is $69.8 \%$. F1 score is represented as:

$F 1=\frac{2}{\frac{1}{\text { recall }}+\frac{1}{\text { precision }}}=2 \cdot \frac{\text { precision.recall }}{\text { recall }+ \text { precision }}$

The final outcome of all the emotions recognized/classified by the Multinomial Naive Bayes are shown in Figure. 10 below.

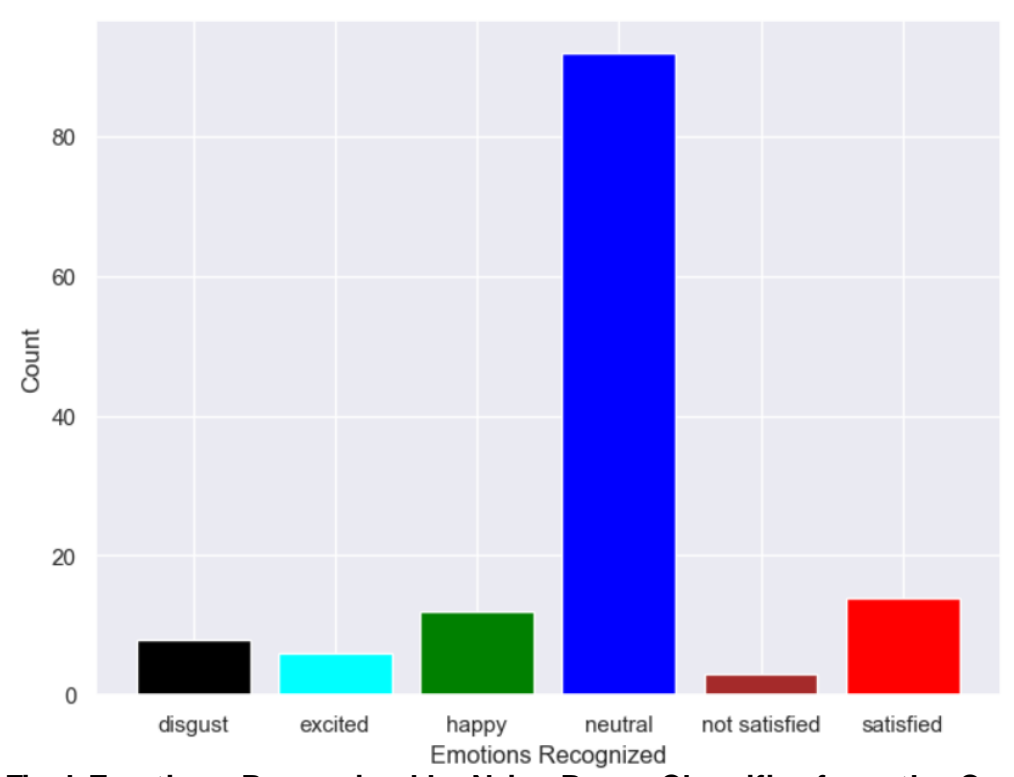

Figure 10. Final Emotions Recognized by Naive Bayes Classifier from the Conversation History of the Chatbot 


\section{Conclusion and Future Scope}

It is essential to understand the sentiment and emotion of the user for evaluating his/her interest in the college and estimating user's future decisions about taking the admission in the college. In this project, we proposed a system where the user can ask their queries in textual or voice-based format to our chatbot system and appropriate response is generated by the chatbot. The proposed methodology classifies emotions of users based on their input queries and also computes the overall sentiment. In the proposed work, data preprocessing is carried out using techniques such as count vectorization and lemmatization. The overall accuracy achieved by the system is $73.8 \%$. In future the accuracy of the proposed system can be increased by combination of feature extraction techniques such as TF-IDF and Count vectorization.

\section{Acknowledgements}

I would like to take this opportunity to thank my internal guide Prof. Jameer Kotwal for giving me all the help and guidance I needed. I am grateful to them for their kind support. Their valuable suggestions were very helpful. I am also grateful to Prof. Archana Chaugule, Head Of Computer Engineering Department, Pimpri Chinchwad College Of Engineering And Research for her indispensable support, suggestions. In the end our special thanks to all the staff members for providing various resources such as a laboratory with all the needed softw are platforms, continuous Internet connections for our project.

\section{REFERENCES}

[1] Ms.Ch.Lavanya Susanna, R.Pratyusha, P.Swathi, P.Rishi Krishna, V.Sai Pradeep," COLLEGE ENQUIRY CHATBOT", International Research Journal of Engineering and Technology (IRJET), on 2020.

[2] Gustavo Assuncao, Paulo Menezes, Fernando Perdigao, "Speaker Awareness for Speech Emotion, Special Focus Paper-Speaker Awareness for Speech Emotion Recognition, 2020

[3] Intelligent ChatBot System using Artificial Intelligence and Deep Learning, International Research Journal of Engineering and Technology (IRJET) on 2020

[4] Chat-Bot”, International Research Journal of Engineering and Technology (IRJET), on 2018

[5] Harimi ${ }^{*}$, A. Shahzadil, A.R. Ahmadyfard and Kh.Yaghmaie Department of Electrical Engineering and Robotics, Shahrood University of technology, Iran09 February 2013 